\title{
Subjective health behaviors in persons suffering from schizophrenia
}

Beata Dutczak, Leszek Bidzan

\section{Source}

Beata Dutczak, Leszek Bidzan. (2010). Subjective health definition and health behaviors in persons suffering from schizophrenia. Ann Gen Psychiatry, vol. 9 (S1)

Persons suffering from schizophrenia as distinct from healthy persons declare more scrupulous behaviors related with control visit to psychiatries and listening their suggestions. Moreover they declare greater gwillingness to gain more medical informations about their disease (causes and treatment) and avoiding stress and strong emotions. 